\title{
Great expectations
}

For referencing Prentice J. Great expectations. WCET ${ }^{\circledR}$ Journal 2021;41(4):7

DOI https://doi.org/10.33235/wcet.41.4.7

The process for applying for Medline Indexing is challenging and multifactorial. Medline is the National Library of Medicine's (NLM) journal citation database of life sciences and biomedical information. It includes bibliographic information for articles from academic journals encompassing medicine, nursing, pharmacy, dentistry, healthcare and veterinary science.

To be considered for listing in Medline, a journal must first apply to the NLM and pass a set of stringent criteria for the application to be successful. From our perspective this has involved our publisher Greg Paull, Emeritus Editor Dr Elizabeth Ayello and me as editor in reviewing the journal's Aims and Scope, Author Guidelines and Policy and Processes. In addition, we have all provided information on our respective positions and responsibilities to the journal and detailed prior experience in scientific publishing and affiliations with other professional bodies.

As the Author Guidelines have been comprehensively revised, we have decided to publish them in this edition on page 27 . We encourage you all to write for the journal and trust these will be helpful.

In evaluating an application, the NLM's Literature Selection Technical Review Committee (LSTRC) considers a journal's scope, scientific content, quality, importance, editorial policies and subject matter. The LSTRC meets three times a year to review and recommend journals for inclusion in MEDLINE.

There are several favourable factors in our application. These being the WCET ${ }^{\circ}$ Journal adheres to the recommendations of the International Committee of Medical Journal Editors (ICMJE Recommendations) and is listed with the ICMJE accordingly. The broad scope and content of the journal plus publication in five languages. The journal is provided to all members of $\mathrm{WCET}^{\circ}$ as part of their membership. A Hybrid Open Access model exists with the WHAM Evidence Summaries for low- and middleincome countries and Editorials, News and views and reports are freely available.

We are hoping our application will be successful or that we are provided with guidance to continue to improve the quality of journal in preparation for a further future application.

The Editorial Board and reviewers of journal articles play a vital role in supporting the editor to maintain the quality of the journal by critiquing articles submitted or publishing articles. I would like to express my appreciation to all Editorial

Jenny Prentice

PhD, BN, RN, STN, FAWMA
Board members and reviewers for your ongoing assistance. As the Editorial Board continues to evolve it is my pleasure to welcome Professor Dieter Hahnloser, EBSQ Coloproctology, University Hospital Lausanne, Department of Visceral Surgery to the Editorial Board. Professor Hahnloser's experience and expertise in rectal cancer, colorectal surgery, inflammatory bowel disease and education and training will be appreciated. We also offer our congratulations to Denise Hibbert on her successful nomination as President Elect for WCET ${ }^{\circledR}$.

During the past year, as indicated in the Index of articles, there has been a diverse range of topics published in the journal from research to clinical care. I thank all authors for their contributions that have assisted in advancing the knowledge and expertise of clinicians involved in wound, ostomy and continence care. The collaboration with Advances in Skin \& Wound Care further enhances this aim. We are hopeful of providing more book reviews in the future and continuing the dialogue on synthesising evidence.

COVID-19 has had unforeseen worldwide ramifications on all aspects of our lives. In the current issue Aydın Ai et al add to the journal's coverage of the effect of COVID-19 on nurses by discussing the frequency and factors around hand dermatitis among nurses during the COVID-19 pandemic. The world has great expectations that the science of vaccines will modify or eradicate this deadly virus. Antimicrobial resistance and antibiotic stewardship is also a global issue. Ousey and Sussman provide an opinion on and highlight the role that pharmacists can play in supporting clinicians to reduce antimicrobial resistance. From a clinical perspective and within their case study Tan NM et al demonstrate how hydrocolloid protective sheets can be used successfully to protect skin integrity from body secretions. Malodourous wounds are challenging to manage. The WHAM evidence summary presents current evidence around the use of yoghurt as a low cost option for managing malodorous wounds.

A subject not often discussed is the impact of illness on sexuality. Sexuality and IIIness - A guidebook for Health Professionals authored by Anne Katz has been reviewed by Harris-Allsop who suggests the book is relevant to the interdisciplinary team as well as being a useful reference for schools of nursing and medicine to guide discussion on illness, sexuality and sexual dysfunction.

It is with great expectation that 2022 will be a year less fraught with global turmoil. In the interim, we wish you all a wonderful festive season and good health.

With Kind Regards

Jenny 